\title{
Postponement of Cell Division by Nutritional Shift-up in Escherichia coli
}

\author{
By FRANÇOIS KEPES* AND THE LATE ADAM KEPES \\ Laboratoire des Biomembranes, Institut Jacques Monod, CNRS, Université Paris VII, Tour 43, \\ 2 place Jussieu, 75251 Paris Cedex 05, France
}

(Received 18 July 1984; revised 23 October 1984)

\begin{abstract}
Nutritional shiftsup of synchronizedor random populations of Escherichia coli (strain ML30 and derivatives of $B / r$ and $K 12$ ) to a richer medium were followed (nutritional pulse) or not (shift-up) by shifts down to the original poor medium. In most cases, the first postshift division was clearly postponed by a shift-up or a nutritional pulse. This delay of the first division was almost constant, whatever the cell age at the time of the transition, and, for the nutritional pulse only, whatever the time spent by cells in the rich medium (between 2 and $25 \%$ of the generation time characteristic of poor medium). Following a shift-up, the new steady state of division rhythm and of mean cell mass was reached at about the second postshift division, whereas after a nutritional pulse, it took three generations to return to the steady state prevailing in poor medium. When both the poor and the rich medium were varied, the extent of the postponement of cell division after a nutritional pulse increased when the amplitude of the stimulus (i.e. the difference in richness) was increased. This was not the case with a simple shift-up, where it seemed that the postponement was compensated in part by the accelerating effects of the rich medium.
\end{abstract}

\section{INTRODUCTION}

Upon transition of a population of bacteria to higher growth rates, complex changes of macromolecular synthesis and cellular parameters occur (Kjeldgaard et al., 1958; Kjeldgaard, 1961 ; Sud \& Schaechter, 1964; Maaløe \& Kjeldgaard, 1966; Helmstetter et al., 1968; Clark, 1968; Cooper, 1969; Sloan \& Urban, 1976). The rate of mass synthesis increases soon after such a shift-up, beginning with ribosome synthesis (Schleif, 1967; Pace, 1973; Shepherd et al., 1980) whereas the rate of cell division increases only 60-70 min later. This 'rate maintenance' is in keeping with the $(I+C+D)$ model of cell division (Cooper \& Helmstetter, 1968; Helmstetter et al., 1968) where $I$ is the interinitiation time, $C$ is the chromosome replication period, followed by the $D$ period which ends at the next cell division. According to this model, only $I$ is regulated in proportion to the growth rate, so that the shift-up would cause only a higher rate of initiation of DNA replication and this would end up with a higher rate of cell division only after a $C+D$ period. This would not occur before about $60 \mathrm{~min}$ (the $C+D$ period) since $C$ and $D$ are largely independent of nutritional status. If the nutritional shift-up can also influence and speed up the rate of DNA replication and/or the processes which span the $D$ period, one may expect a somewhat earlier increase in the rate of cell division. This was observed by Sloan \& Urban (1976) upon passage of Escherichia coli $15 \mathrm{~T}^{-}, \mathrm{B} / \mathrm{r}$ or K12 from a poor medium supporting a $120 \mathrm{~min}$ generation time to a glucose medium. Loeb et al. (1978) found that the perturbation was different in the two substrains of $E$. coli $\mathrm{B} / \mathrm{r}$ they used, and was dependent upon the age of the cells at the time of the shift-up. Contrary to the findings of Sloan \& Urban (1976) with a B/r strain, these authors also showed that the postshift rate of cell division during the first hour was equal to or less than the preshift rate, provided that the latter was slow. Many observations have supported the idea that the rate of DNA synthesis after a shift up maintains its preshift level for about $20 \mathrm{~min}$, before it accelerates (Kjeldgaard et al., 1958; Cooper, 1969). More recent data suggest 
that DNA synthesis in a K12 strain is inhibited during the first 25 min after a shift-up (Zaritsky \& Zabrovitz, 1981).

The method of synchronization described by Kepes \& Kepes (1980) provided a new opportunity of re-examining this question. In order to observe the actual inhibitory influences of a shift-up uncomplicated by expected accelerating effects of the rich medium on some cell processes, we carried out a second type of experiment, in which a culture was briefly exposed to broth and then returned to its original poor medium (nutritional pulse). Experiments were also done to confirm that the behaviour of exponentially growing cultures did not contradict the surprising kinetics observed in the synchronous populations.

\section{METHODS}

Bacteria. The strains of $E$. coli used were: $\mathrm{ML} 30\left(\mathrm{Mel}^{-} \mathrm{Lac}^{+}\right.$), derived from ML3; GC4506 (Ura- $\Delta l a c \mathrm{Str}^{\mathrm{r}} \mathrm{Km}^{\mathrm{r}}$ (Tn5 : :pyrD)) derived from K12 594; and GC1055 (Mal ${ }^{-} \mathrm{Lac}^{+} \mathrm{Gal}^{+}$), derived from B/r. Strains GC4506 and GC1055 were provided by R. d'Ari, Institut J. Monod, Paris, France. Strain ML30 is from the Institut Pasteur, Paris, France.

Media. Bacteria were grown aerobically in conical flasks in a minimal medium containing, per litre of distilled water: $10 \mathrm{~g}$ MOPS (Calbiochem-Behring), $4 \mathrm{~g}$ glycerol, $4.6 \mathrm{~g} \mathrm{NaCl}, 1.5 \mathrm{~g} \mathrm{KCl}, 2 \mathrm{~g}\left(\mathrm{NH}_{4}\right)_{2} \mathrm{SO}_{4}, 0.2 \mathrm{~g}$

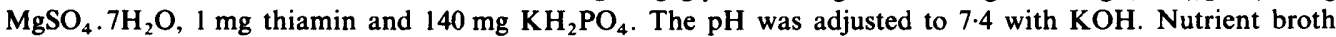
contained, per litre of distilled water : $5 \mathrm{~g} \mathrm{NaCl}, 10 \mathrm{~g}$ Bacto-Tryptone (Difco) and $5 \mathrm{~g}$ yeast extract (Difco). The pH was adjusted to 7.4 with $\mathrm{NaOH}$. Preconditioned broth was nutrient broth in which E. coli was grown to an $\mathrm{OD}_{600}$ of about 0.02 and then removed by filtration.

Nutritional shifts. Shift-up experiments were performed by dilution of a portion of the culture into prewarmed nutrient broth. A nutritional shift-down was obtained by collecting cells from nutrient broth by centrifugation (strain GC1055) or on a pre-rinsed membrane filter (HA 0.45, Millipore), washing them, and resuspending them in the previous minimal medium. This operation required about $1 \mathrm{~min}$ and the delay of the next division due to this procedure without a nutritional shift was measured to be $3-5 \mathrm{~min}$.

Synchronization procedure. Cells were synchronized by repeated short phosphate starvations followed by feeding, in a phosphate limited medium. This method has been extensively described elsewhere (Kepes \& Kepes, 1980).

Optical density measurements. Optical density was measured at $600 \mathrm{~nm}$ in a Zeiss PM6 spectrophotometer.

Cell counting. Bacterial concentrations were determined with a Coulter counter (model ZB, Coultronics France), equipped with a $50 \mu \mathrm{m}$ orifice, in samples diluted 100- to 1000-fold in filtered saline (Isoton II, Coultronics France). Cell concentrations are shown in arbitrary units on the figures. These concentrations were kept below 4 $\times 10^{8}$ cells $\mathrm{ml}^{-1}$ in minimal medium and $4 \times 10^{7}$ cells $\mathrm{ml}^{-1}$ in broth for all experiments.

\section{RESULTS}

\section{Shift-up and nutritional pulse of exponential-phase populations}

Cultures of each of the three strains used were grown exponentially in glycerol minimal medium. They were diluted threefold into prewarmed nutrient broth at time zero, and either left in broth (shift-up) or shifted down after $0 \cdot 1$ generation time (characteristic of the poor medium) by transfer back into the original minimal medium (nutritional pulse). Cell concentrations were measured in the shifted, pulse-shifted and unshifted cultures. With all three strains, the optical density of the upshifted culture increased at its postshift rate after a 7-10 min extrapolated lag, or was essentially unperturbed by the nutritional pulse (data not shown). The patterns of cell division were reproducible for a given strain, but differed significantly from one strain to another. The results are shown in Fig. $1(a-c)$, the upper curves showing the pattern of cell division obtained after a simple shift up and the lower curves showing the pattern after a nutritional pulse.

Fig. $1(a)$ represents the results obtained with the $E$. coli $\mathrm{B} / \mathrm{r}$ strain, GC1055. The pattern following a shift-up in this strain was the closest to the classical 'rate maintenance', with some slight deviations. The extrapolated duration of rate maintenance, i.e. the time elapsed from the shift to the intercept of the two steady-state straight lines, was around $60 \mathrm{~min}$. The maximal deficit of cell number compared to the control occurred at about $50 \mathrm{~min}$ and amounted to approximately $10 \%$. After a nutritional pulse, the B/r strain exhibited a total inhibition of cell division for about $30 \mathrm{~min}$. This led to a $35 \%$ maximal deficit of cell number before division 


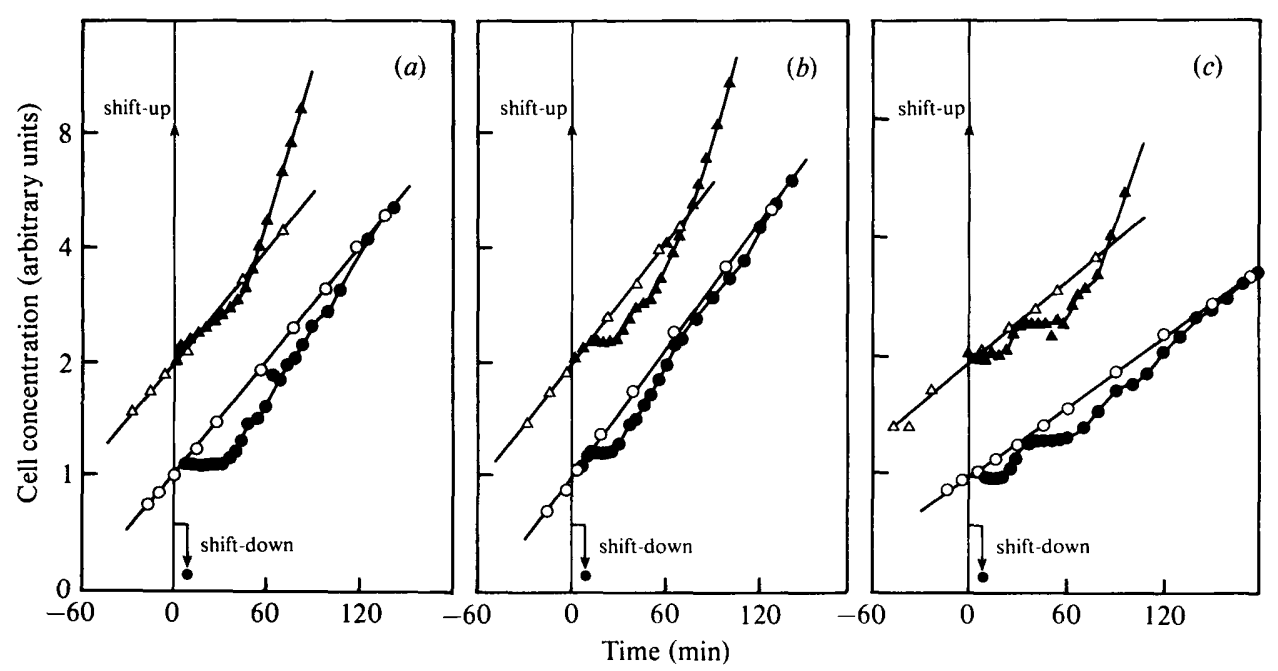

Fig. 1. Nutritional shifts of exponential-phase populations of $E$. coli strains GC1055 (B/r derivative) (a), ML30 (b) and GC4506 (K12594 derivative) (c). Bacterial populations growing exponentially in a glycerol minimal medium (open symbols) were diluted threefold at zero time in nutrient broth (closed symbols). They were either left in broth (triangles) or shifted down $0 \cdot 1$ generation time (characteristic of poor medium) later (circles). For the $B / r$ strain only, the downshift involved centrifugation instead of filtration. Cell concentrations are plotted versus time on a semi-logarithmic plot. These experiments were done at least twice, with very similar results.

resumed. The response of strain ML30 to a shift-up (Fig. $1 b$ ) can be described as rate maintenance for about $10 \mathrm{~min}$, followed by $20 \mathrm{~min}$ inhibition of cell division. After an intermediate period, the bacterial concentration increased at the rate supported by the richer medium. The intercept between straight-line extrapolates occurred at about $70 \mathrm{~min}$ and the maximal deficit of cell number compared to the control was $20 \%$, at $50 \mathrm{~min}$. The cell division pattern of strain ML30 for about $1 \mathrm{~h}$ following the nutritional pulse looked very much like its pattern after a shift-up, except for the final acceleration in the rich medium. The kinetics of cell division of strain GC4506 (a derivative of K12 594) upon shift-up (Fig. 1c), in contrast to the B/r strain and strain ML30, exhibited an immediate inhibition followed after 18-24 min by a burst of division of some $25 \%$ of the population. Another plateau was then observed for about $30 \mathrm{~min}$ before cell division resumed. The postshift rate of division was obtained about 70 min after shift up. The maximal deficit of cell number occurred at $55 \mathrm{~min}$ and amounted to $15 \%$. For about $1 \mathrm{~h}$ after the shift, the division patterns of the K12 594 strain subjected to a nutritional pulse or to a shift-up looked very much alike. After these initial responses to the nutritional pulse stimulus, all three strains showed a slow return of the cell number towards the control steady state, which was attained substantially later than the steady state in the simple shift-up experiments.

The experiments reported below were done with synchronized populations of E. coli ML30, since it exhibited the most conspicuous deviation from the classical response to nutritional shiftup, and also the simplest pattern.

\section{Shift-up of synchronized populations}

After 14 phasing cycles, a culture of E. coli ML30 was diluted 60-fold in glycerol minimal medium and allowed to grow in non-limiting conditions. Four portions of this culture were diluted fourfold into broth at different stages of the third and fourth cell cycle. The postshift cell counts and the counts in the unshifted culture are given in Fig. 2. The doubling time for optical density changed from about $60 \mathrm{~min}$ in minimal medium to $20 \mathrm{~min}$ in the rich medium. Given that the transfers were done $17 \mathrm{~min}$ apart, the successive curves of optical density or of cell concentration in broth were expected to be about $11 \mathrm{~min}$ apart. The experimental data fit this prediction well two generations or more after shift when the new steady state had been reached and the mean cell mass had increased by a factor of about 5-6, but, interestingly, the first 


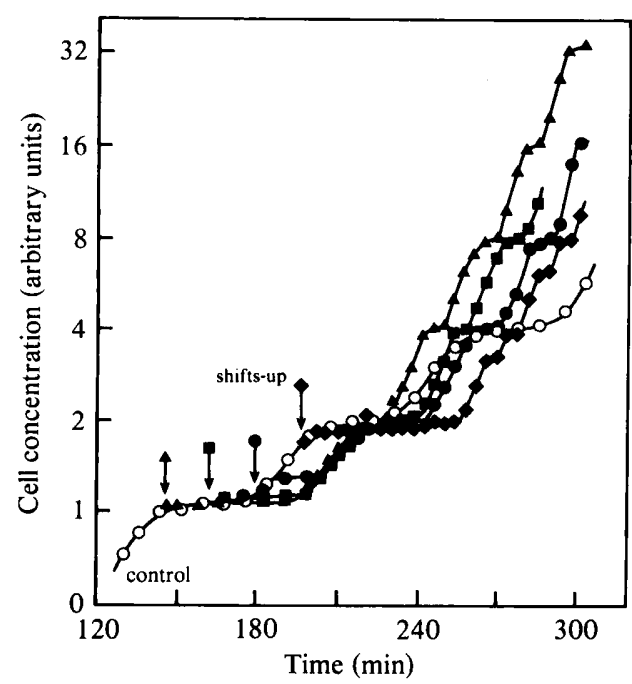

Fig. 2. Nutritional shifts of a synchronized culture. A culture of $E$. coli ML30 was phased through 14 artificial cycles and then allowed to grow in non-limiting conditions by a 60 -fold dilution in glycerol minimal medium at time zero (open symbols). More than two generations later, four samples were consecutively diluted fourfold into nutrient broth (arrows and closed symbols). Cell counts were corrected for dilution and are slightly transposed along the vertical axis for clarity. The experiment shown is representative of six separate experiments.

division in all four shifted cultures took place about $20 \mathrm{~min}$ after the division in the control culture, all within a period of $6 \mathrm{~min}$. The use of preconditioned broth and of different dilution factors ranging from 1.4 to 20 did not alter these results. A moderate shift-up by addition of a negligible volume of glucose plus Casamino acids to a synchronous culture of $E$. coli ML30 also triggered a delay of the first division (data not shown). The observed 20 min inhibition of division was consistent with that observed in exponential-phase populations (Fig. $1 b$ ). Nevertheless, when the shift-up was done close to mid-division (e.g. third transfer in Fig. 2), part of the population escaped inhibition and underwent division in the next few minutes. This phenomenon may account for the $10 \mathrm{~min}$ apparent rate maintenance seen in the experiment done with exponentially growing strain ML30 (Fig. $1 b$ ).

It is noteworthy that the first postshift division, which was delayed, was spread in time to the same extent as in the slow-growing control, whereas the following divisions were more narrowly synchronous, approximately in proportion to the shortening of the doubling time in nutrient broth.

In contrast with the coincidence of the first division whatever the cell age at the time of shiftup, the second postshift division occurred after a variable delay such that the cell had time to attain the steady-state mass characteristic of the rich medium. The interdivision time for subsequent divisions remained constant, characteristic of the steady state in rich medium. The regularity of these events appears clearly in Fig. 3, which was constructed from the data of Fig. 2 and several similar experiments. In Fig. 3(a), once the abscissa, i.e. the cell age at the time of shift, is defined, the time of occurrence of the subsequent cell divisions can be predicted along the corresponding vertical line. The cell age is given in min and also as a fraction of a generation time. The near constancy of the delay in the first (but not the second) postshift division is shown in Fig. 3(b). The delay values increase from 15 to $24 \mathrm{~min}$ as a function of cell age at the time of shift up.

\section{Nutritional pulse of synchronized populations}

In view of the 'all-or-none' retardation of cell division by about $20 \mathrm{~min}$, even after passage into nutrient broth in the very late part of the cell cycle, the question arose of how much time was necessary to trigger this effect. It was clear that 0.1 generation time was sufficient (Fig. $1 b$ ). To 



Fig. 3. Cell divisions as a function of cell age at the time of shift-up. (a) The time elapsed from shift-up to the following mid-divisions in six separate experiments with $E$. coli ML30 is plotted versus cell age at the moment of the shift. (b) The time elapsed from the reference division in the unshifted culture to the first and second mid-divisions after the shifts is plotted versus cell age at the moment of the shift (same experiments as in $a$ ). In both $(a)$ and $(b)$, straight lines were drawn from linear regressions.

explore this further, synchronized cultures were diluted into broth and shifted back into the original minimal medium after various short intervals. In the experiments shown in Fig. 4, samples of a synchronously growing culture of $E$. coli ML30 were transferred into broth at onethird of the second cell cycle following synchronization, for 1,6 or $12 \mathrm{~min}$, before being shifted down to their original medium by filtration and resuspension. Cell concentration and optical density were measured in these cultures. A transient inhibition of about 20 min was again observed for the first division of all subcultures compared to the control population. Since the mass increase was only slightly accelerated during the short exposure to broth, the return to steady state would mean identical cell concentration curves within experimental error. In fact it took two further cell cycles to approach this steady state in two steps (Fig. 4), as already suggested by the results shown in Fig. $1(b)$.

In other experiments, nutritional pulses of constant duration were applied at different phases of the cell cycle, and again a uniform retardation of about $20 \mathrm{~min}$ of the next cell division was observed. The results of experiments involving nutritional pulses on synchronized cultures (Table 1) show that the delay of cell division was almost constant whatever the age at shift up or the time spent in broth.

In a last series of experiments, the amplitude of the stimulus (i.e. the difference in richness) was varied by changing both the poor medium and the richer medium in which $E$. coli ML30 spent 0.1 generation time (characteristic of the poor medium). Table 2 summarizes these results and gives for each pair of media the extent of the delay observed for the first postshift division. 


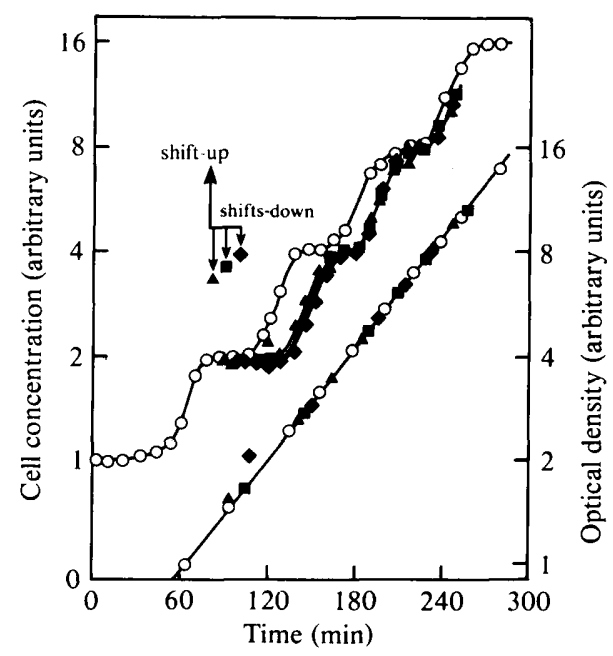

Fig. 4. Effect of short exposures to nutrient broth of a synchronized culture. After 16 phasing cycles, a culture of $E$. coli ML30 was diluted at time zero and grown in non-limiting conditions (open symbols). At the beginning of the second cell cycle (arrow up), samples of the culture were diluted fourfold into broth, and 1, 6 or 12 min later (arrows down), the samples were shifted down (closed symbols) back to minimal medium. Cell counts and optical density measurements were corrected for dilution. A single straight line was drawn through all the experimental points of optical density. The experiment shown is representative of four separate experiments.

\section{DISCUSSION}

The cell division responses obtained after a shift-up or nutritional pulse in exponential-phase populations of E. coli ML30 are compatible with the corresponding responses in synchronous populations obtained by repeated phosphate limitations. This synchronization procedure is therefore a valid method for use in studies of nutritional transitions, and moreover makes the observations easier and more accurate than with less sharply synchronized or random populations.

E. coli ML30 and the K12 594 strain showed a delay of their first cell division following a shiftup from glycerol minimal medium to broth. This delay is not linked to any particular element of the complex medium, like salt, since the addition of glucose, or glucose plus Casamino acids, in a negligible volume, also led to a transient inhibition of division. We suggest the term 'postponement' of cell division. The term 'transient inhibition' of cell division is unsatisfactory since we could not detect any dose-effect relationship between the retarding influence and its result, and since the inhibitor (the increased nutrient value) can be absent at the time when inhibition occurs (nutritional pulses).

Shift-up experiments have been commonly interpreted in terms of the near constancy of the $C$ and $D$ periods, and deviation from the rate maintenance of cell division has been explained by variations of these periods upon a shift-up (Cooper, 1969; Sloan \& Urban, 1976; Loeb et al., 1978). These interpretations are made more difficult to accept by the observation that DNA synthesis is inhibited during the first $25 \mathrm{~min}$ after a shift-up (Zaritsky \& Zabrovitz, 1981). Another key to the understanding of our data and of previous data can be found in Fig. $1(a)$. The B/r strain GC1055 shows approximately the classical rate maintenance after a shift-up but, interestingly, its rate of division slows down sharply if this shift-up is soon followed by a shift back to minimal medium (nutritional pulse). A strain thus responds to a shift-up by a basic postponement of its next division, more or less compensated by the accelerating effects of the rich medium. These accelerating effects are suppressed in our nutritional pulse experiments. Our results are discussed below, keeping in mind the contradictory effects of a shift-up.

The first division following a shift-up in E. coli ML30 was postponed by a constant amount of time, whatever the cell age at the time of the shift (Figs 2 and 3). Loeb et al. also found such a 
Table 1. Nutritional pulses in synchronized populations of E. coli ML30

The results are from experiments in which the cell age at pulse or the length of the pulse were varied. The cells were grown in a glycerol minimal medium and transferred to broth for the time shown.

$\begin{array}{cc}\begin{array}{c}\text { Cell age at } \\ \text { shift-up } \\ \text { (fraction of } \\ \text { generation time) }\end{array} & \begin{array}{c}\text { Time spent } \\ \text { in broth } \\ \text { (min) }\end{array} \\ 0.33 & 12 \\ 0.49 & 12 \\ 0.66 & 12 \\ 0.83 & 12 \\ 1 & 12 \\ 0.25 & 5 \\ & 10 \\ 0.51 & 5 \\ & 12 \\ 0.31 & 1 \\ & 6 \\ 0.50 & 12 \\ & 3 \\ 0.26 & 12 \\ 0.64 & 3 \\ & 3\end{array}$

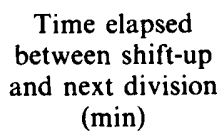

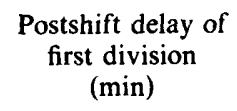

Table 2. Nutritional pulses in synchronized populations of E. coli ML30 with various shift amplitudes

The results are from experiments involving nutritional pulses where both the poorer and the richer medium were varied. Each value of the extent of the division delay is the mean of at least three distinct measurements, and was corroborated by at least one experiment using exponential-phase populations. The generation times supported by a given medium were measured in steady state.

Poorer medium*

Alanine + Proline

Glycerol

Glucose
Richer medium $\dagger$

Glucose
Glucose + Casamino
acids
Nutrient broth
Glucose
Glucose + Casamino
acids
Nutrient broth
Glucose + Casamino
acids
Nutrient broth

\section{Ratio of} generation times

$0 \cdot 5$

$0 \cdot 33$

$0 \cdot 25$

0.75

0.5

0.38

0.63

0.5
Postshift delay of the first division (min)

10

26

32

6

18

15

* Alanine, proline, glycerol and glucose were each used at $0 \cdot 4 \%$ (final concentration).

+ Glucose and Casamino acids were used at $0.4 \%$ (final concentration). Nutrient broth is described in Methods.

postponement with $E$. coli strain $\mathrm{B} / \mathrm{r} \mathrm{F}$ subjected to a similar shift-up, but in their experiment the extent of the early perturbation was dependent on the cell age. Clark (1968) reported that the first and third generation times of $E$. coli $\mathrm{B} / \mathrm{r}$ were independent of cell age after a shift from a $47 \mathrm{~min}$ doubling time to $23 \mathrm{~min}$. He observed, as we did, that only the second generation time was dependent on the cell age at the time of the shift. He also described the effects of a shift of a culture growing on acetate ( $140 \mathrm{~min}$ doubling time) to a $23 \mathrm{~min}$ doubling time. The first division was either unaffected, for cells shifted late in their cycle, or speeded, in cells shifted early. Our experiments with alanine plus proline as carbon sources ( $94 \mathrm{~min}$ generation time) and dilutions in broth have confirmed Clark's observations (data not shown) but it can be seen from Fig. 4 and 
Table 2 that, using the same media, a nutritional pulse leads to a constant postponement whatever the cell age. This comparison can be taken as another illustration of the contradictory effects of a simple shift.

After a shift-up, the new steady state of division rhythm and of mean cell mass is almost reached at the time of the second postshift division (Clark, 1968; Fig. 3 of this work). These results do not support the finding of Sloan \& Urban (1976) with E. coli $15 \mathrm{~T}^{-}$, that the new steady cell mass was still not reached $10 \mathrm{~h}$ after a shift from an aspartate to a glucose medium.

Maaløe \& Kjeldgaard (1966) were, to our knowledge, the first authors to describe 'doubleshift' (nutritional pulse) experiments. They measured only optical density. One of their conclusions was that the time spent in broth should be kept below $15 \mathrm{~min}$ in order not to observe a lag after the shift-down. They attributed the lag to the repression in broth of the synthesis of enzymes required in minimal medium. Accordingly, the bacteria spent no more than $12 \mathrm{~min}$ in rich medium in our pulse-shift experiments. The quality of synchrony in our cultures remained sufficient through a double-shift to allow us to describe the following division pattern. It appeared that the postponement of the first postshift division was substantially the same whatever the duration of the exposure to the rich medium, from $1 \mathrm{~min}$ up. The latter contention has an inherent inaccuracy, since $(a)$ the time of filtration, washing and resuspension, which terminates the exposure, is not completely controlled, and $(b)$ some metabolic pools replenished in rich medium could take some time to readjust to the steady state prevailing in poor medium.

The extent of the postponement of cell division after a nutritional pulse increased when the amplitude of the stimulus was increased (Table 2). If one takes into account the accelerating effects of a rich medium after a simple shift, this conclusion is qualitatively in agreement with the data of Clark (1968) and of Loeb et al. (1978).

Since the postponed division after a nutritional pulse is expressed in the absence of the effector (the increased supply of nutrients), an important feature of these short exposure experiments is in the storage of the information concerning the next cell division. This expression of a kind of cell 'memory' is certainly worth exploration. Apart from its intrinsic interest, the nutritional pulse can be considered as a tool to study the transient inhibition of division, since for instance labelling studies before and after a nutritional pulse can be done in comparable conditions.

Professor Adam Kepes passed away suddenly on April 18, 1984. This article is dedicated to the memory of Adam Kepes whose insight and inexhaustible enthusiasm stimulated the work of his collaborators each day until that fatal day.

\section{REFERENCES}

ClARK, D. J. (1968). The regulation of DNA replication and cell division in Escherichia coli $\mathrm{B} / \mathrm{r}$. Cold Spring Harbor Symposia on Quantitative Biology 33, 823-838.

COOPER, S. (1969). Cell division and DNA replication following a shift to a richer medium. Journal of Molecular Biology 43, 1-11.

COOPER, S. \& Helmstetrer, C. E. (1968). Chromosome replication and the division cycle of Escherichia coli B/r. Journal of Molecular Biology 31, 519-540.

Helmstetter, C. E., Cooper, S., Pierucci, O. \& REVELAS, E. (1968). On the bacterial life sequence. Cold Spring Harbor Symposia on Quantitative Biology 33, 809-822.

KEPES, F. \& KEPES, A. (1980). Synchronisation automatique de la croissance d'Escherichia coli. Annales de microbiologie 131 A, 3-16.

KJeldgaARD, N. O. (1961). The kinetics of ribonucleic acid and protein formation in Salmonella typhimurium during the transition between different states of balanced growth. Biochimica et biophysica acta 49, 64-73.

KJeldgaArd, N. O., MaAløe, O. \& Schaechter, M. (1958). The transition between different physiological states during balanced growth of Salmonella typhimurium. Journal of General Microbiology 19 , 607-616.

Loeb, A., McGrath, B. E., Navre, J. M. \& Pierucci, O. (1978). Cell division during upshifts of Escherichia coli. Journal of Bacteriology 136, 631-637.

MaAløE, O. \& KJeldgaARD, N. O. (editors) (1966). Control of Macromolecular Synthesis. New York: W. A. Benjamin.

PACE, N. R. (1973). Structure and synthesis of the ribosomal ribonucleic acid of prokaryotes. Bacteriological Reviews 37, 562-603.

SCHLEIF, R. (1967). Control of production of ribosomal protein. Journal of Molecular Biology 27, 41-55.

SHEPHERD, N., CHURCHWARD, G. \& BREMER, $H$. (1980). Synthesis and function of ribonucleic acid 
polymerase and ribosomes in Escherichia coli $\mathrm{B} / \mathrm{r}$ after a nutritional shift-up. Journal of Bacteriology 143, 1332-1334.

Sloan, J. B. \& Urban, J. E. (1976). Growth response of Escherichia coli to nutritional shift up. Journal of Bacteriology 128, 302-308.

Sud, I. J. \& Schaechter, M. (1964). Dependence of the content of cell envelopes on the growth rate of Bacillus megaterium. Journal of Bacteriology 88, 1612-1617.

ZARITSKY, A. \& ZABROVITZ, S. (1981). DNA synthesis in Escherichia coli during a nutritional shift-up. Molecular and General Genetics 181, 564-566. 